\title{
SWING LOAD STABILIZATION FOR MINING AND CONSTRUCTION APPLICATIONS
}

\author{
Peter I. Corke* Jonathan M. Roberts* \\ Graeme J. Winstanley* \\ * CSIRO Manufacturing Scicnce \& Technology, \\ CRC for Mining Technology \& Equipment, \\ PO Box 883, Kenmore, Qld 4069, Australia. \\ email: $\{$ pic,jmr,gjw\}@cat.csiro.au. \\ http://www.cat.csiro.au/cmst/automation
}

\begin{abstract}
This paper discusses the issue of sensing and control for stabilizing a swinging load. Our work has focussed in particular on the dragline as used for overburden stripping in open-pit coal mining, but many of the principles would also be applicablc to construction cranes. Results obtained from experimental work on a full-scale production dragline are presented.
\end{abstract}

Keywords: dragline, crane, control, load stabilization, mining, construction

\section{INTRODUCTION}

Crane type machincs are used for a wide variety of material handling problems in mining, construction and cargo handling. 'The load may' be suspended by one or more ropes from a trolley that may move in one or two dimensions, or from a rotating house. Construction cranes typically have a single hoist rope and a rotating cab, the dragline, see Figure 1, has a hoist 'and drag rope, a fixed boom and a rotating house on a walking base.

Compared to conventional robotic devices cranes can have a very large working cnvelope, however they lack rigidity and the load is free to swing. In general these machines are manually operated and require significant operator skill in order to manage, and even exploit, the swing of the load.

In order for an automatic control system to perform the same task as the operator it is necessary to have both appropriate sensors and a dynamical model of the swinging load. These topics are covered in sections 3 and 4 respectively, and 5 presents conclusions.

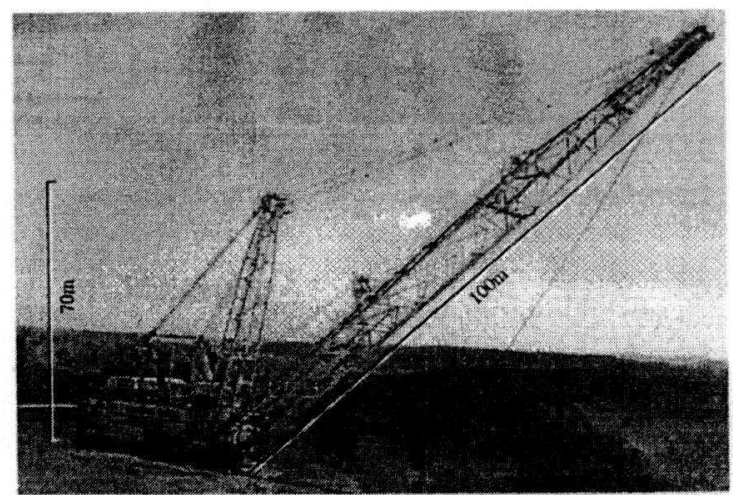

Fig. 1. A relative of the construction crane - the dragline excavator.

\section{PREVIOUS WORK}

There is considerable literature in topics such as crane load and helicopter load stabilization. The crane literature is largely focussed on open-loop, or input shaping, approaches rather than loadposition feedback. If the crane velocity follows a trapezoidal profile, where the ramp times are equal to the period of the swinging load, then an initially unswinging load will be left with no 

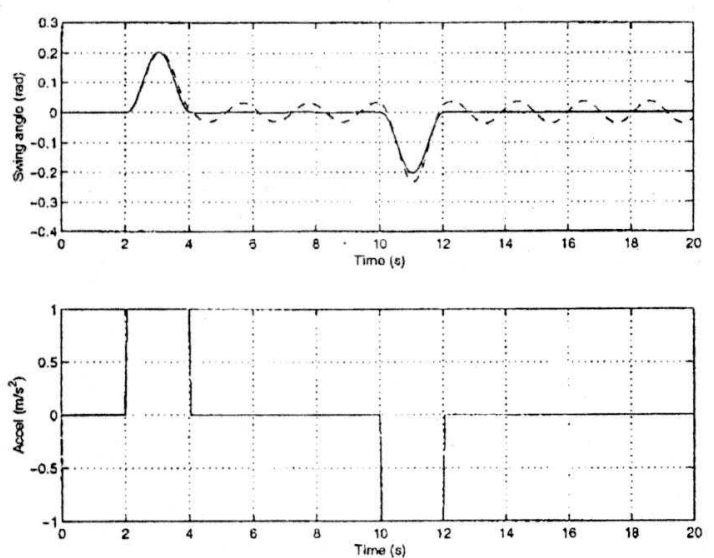

Fig. 2. Simulation of open-loop anti-sway crane motion strategy. Acceleration time is equal to swing period. The solid line corresponds to the case where swing period is accurately known, the dashed line corresponds to a $10 \%$ change in rope length that is not known to the controller.

swing, see Figure 2. However an error in the rope length used will result in imperfect swing elimination. A variant of this[1] is discontinuous swing control which uses maximum acceleration and deceleration rates and gives motion times comparable to time-optimal strategies. Such strategies are the basis of a number of anti-swing or anti-sway motion controllers.

Backhouse et al.[2] argue that with modern highquality velocity controlled drives there is no need for load position feedback, and in an indoor windgust free environment this solution may be adequate. Feddema[3] describes an input shaping technique for flexible manipulators and suspended loads. A digital filter is interposed between the operator and the crane so that residual swing is reduced. The filtcr dynamics are dependent on the swinging load dynamics and must be adjusted online. However like all open-loop techniques this approach cannot reject external disturbances.

More recently DeSantis and Krau[4] discuss bangbang control of a Cartesian crane, but present only simulation results for the simple case where rope length remains constant. The bang-bang controller did not appear to be significantly better than the state-feedback controller given for comparison.

Ridout[1] discusses closed-loop control based on feedback of trolley position and velocity and load angle. While suboptimal in terms of motion time the approach was shown to be very robust with respect to changes in load and rope length. This controller was extended to incorporate variable damping[5]. Variable damping is based on the characteristics of a lightly damped system which has a fast rise time (desirable) but considerable

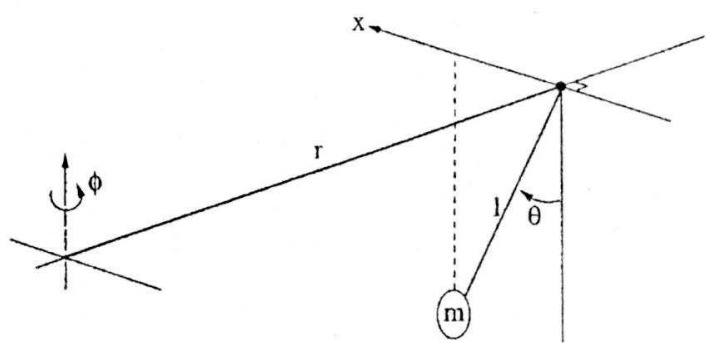

Fig. 3. Notation for simplified pendulum case.

overshoot (undesirable). If the damping is increased as the load approaches the setpoint the system can be made to settle quickly.

Various approaches to load position sensing have been proposed. Ridout[1] uses a small lever arm contacting the rope. Others have proposed to measure the force exerted on the trolley by the load, but in practice these measurements are noisy and corrupted by friction. For large cranes, such as used in civil construction or ports, it may be feasible to instrumert the spreader with sensors.

There is also considerable literature related to helicopters carrying large loads $[6,7]$. The similarity to the dragline problem is that the load mass is a significant fraction of the vehicle mass - for a dragline the load inertia is a significant fraction of house rotational inertia. The helicopter literature is largely concerned with the dynamics of the interaction between the suspended load and the craft's aerodynamic model, which results in an open-loop unstable system. A skilled pilot can control this system but with increased workload. Stabilization involves feedback of vehicle and load relative attitude. The latter is obtained from the displacement of a spring mounted ring through which the suspension cable passes. The non-linear dynamics are linearized and an optimal state feedback controller and state estimator are designed.

\section{LOAD SWING CONTROL}

\subsection{Fundamentals of pendulum stabilization}

The principle behind the concept of dragline bucket swing control is quite straightforward. Consider the simplified situation of Figure 3 where the pendulum is suspended from an arm rotating about a vertical axis. The linearized equation of forced motion is

$$
\ddot{\theta}=-\frac{g}{l} \theta-\frac{\ddot{x}}{l}
$$

where $\ddot{x}$ is the the linear acceleration of the pendulum's suspension point, and is related to the rotational acceleration of the arm by

$$
\ddot{x}=\ddot{\phi} .
$$



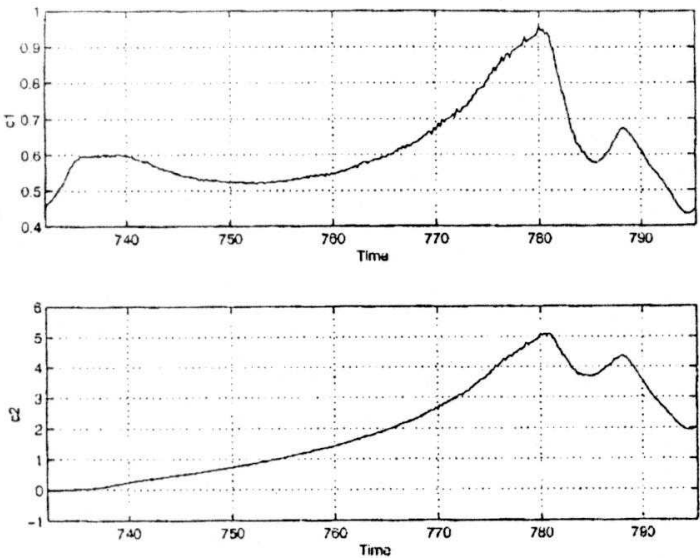

Fig. 4. Variation of equivalent pendulum coefficients over a typical cycle.

The linearized equation of motion can now be written as

$$
\ddot{\theta}=-\frac{g}{l} \theta-\frac{r}{l} \ddot{\phi}
$$

which shows that the pendulurn motion responds to the rotational acceleration of the arm which is directly related to the torque applied to the slew drive. This is why a dragline's slew drive is current (or torque) controlled rather than speed controlled like the hoist and drag drives.

Now consider a fcedback controller where the applied torque is

$$
\tau=P \theta+D \dot{\theta}
$$

and the motion of the arm is undamped

$$
\ddot{\phi}=\frac{\tau}{J}
$$

with rotational inertia $J$. The closed-loop dynamics become

$$
\ddot{\theta}+\frac{r D}{l J} \dot{\theta}+\left(\frac{g}{l}+\frac{r P}{l J}\right) \theta=0
$$

from which it can be seen that the closed-loop damping is a function of $D$ and the natural frequency a furction of $P$. That is, making the slew torque proportional to the bucket velocity increases the damping and reduces the oscillation - this would be the essential aspect of the operator's learnt skill.

For the dragline[8], rather than a simple pendulum, we can write

$$
\ddot{\theta}=-c_{1} \theta-\frac{c_{2}}{J_{h}} \tau
$$

where $c_{1}$ and $c_{2}$ are configuration varying parametcrs, whose typical change over a swing cycle are shown in Figure 4. The torque coupling coeflicient, $c_{2}$, in particular varies very significantly.

\section{ROBLST LOAD POSITION SENSING}

Encoders can be fitted to winch drums to give an indication of the payed out rope length. However for loads suspended by one or two ropes there will still be respectively two or one degrees of freedom in which the load is free to swing. Motion along these degrees of freedom must be measured for control purposes.

The performance criteria for such a sensor are stringent, particularly for mining operations, and must be capable of operating 24 hours a day in all weather conditions and at a rate of at least $3 \mathrm{~Hz}$, (control constraint).

For crane applications it may be possible to fit. instrumentation to the load itself but common sensors have drawbacks. Inertial sensors may have velocity offsets that vary with time, and GPS sensors may give errors due to electro-magnetic propagation effects in the vicinity of last metal structures such as the crane itsclf. For a dragline excavator the extremely harsh nature of the bucket's interaction with the ground precludes instrumentation on the bucket itsclf.

At first glance, computer vision appears to be an ideal sensor for this application: the sensors are low cost, processing power is becoming cheaper, it is able to measure the stiate of the load without being in contact, and it mimics the human operator's sensing modality. Experiments with computer vision were reported in $[9,10]$. Strong shadows, sun dazzle, background texture and clutter, moving camera frame, and lack of contrast combined to thwart all the machine vision approaches that we evaluated.

\subsection{A laser based approach}

The machine vision experiments did teach us that hoist ropes may be a easier to locate than the bucket itself. The approach selected for the automation system is instead based on a scanning infra-red laser range-finder that looks for the ropes. This retains the key advantage of noncontact position sensing, the devices are rugged and low in cost, and the difficult problem of robust scene segmentation is side stepped.

Note that for a dragline, as opposed to most cranes, there are actually two hoist ropes separated by about one metre. The angles computed are therefore the angles for an imaginary average hoist rope located mid-way betwcen the left and right hoist, ropes.

Our system uses two Proximity Laser Scanners (PLS) manufactured by Sick Opto-electronics, Germany. The PLS returns range, $R$, and bearing, $\omega$, data across a $180^{\circ}$ field-of-view, giving a 


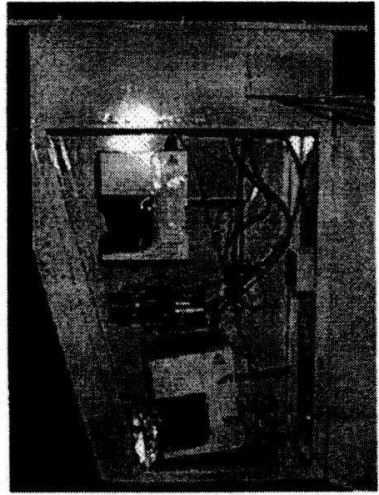

Fig. 5. The sensor package housing showing the two scanners and the camera.

reading every $0.5^{\circ}$ and a range accuracy of $20 \mathrm{~mm}$ and have been extensively tested under the range of conditions expected on a dragline (night, day, rain and dust). Two laser scanners are used for redundancy, and to address the problem of blinding due to direct sunlight. The two scanners have different look-down angles (Figure 5) and so can not be blinded by the sun simultaneously. With some knowledge of the geometry of the dragline boom tip, it is possible to calculate swing and hoist angles dircctly from the laser scanner data.

The raw data from the laser scanners passes to the rope finding element (Figure 6), which extracts the range and bearing of the two ropes with respect to the scanner in the scanning plane (Section 4.1.1). These positions are passed to a transformation element that transforms the positions of the ropes in the scanning plane to swing and hoist angles of the hoist rope. The swing and hoist angles for each rope are averaged, and the average is passed to a Kalman filter. Finally, the Kalman filter produces estimates for swing and hoist angles and the respective velocities (Section 4.2).

4.1.1. Finding the ropes Each laser scanner produces 361 measurements per scan. Each measurement represents the range to the first object in the corresponding $0.5^{\circ}$ segment of the semi-circular scan. The positions of the two hoist ropes must be extracted from this dati. False targets (targets that are not the ropes) complicate the task of tracking the ropes. The rope finding algorithm therefore must be able to locate the positions of the hoist ropes reliably in the presence of these false targets.

4.1.1.1. False targets Rain drops may cause false targets. The laser scanners on the dragline are mounted below the boom structure near the tip of the boom, see Figure 1. The extent to which rain drops can be seen in this region,

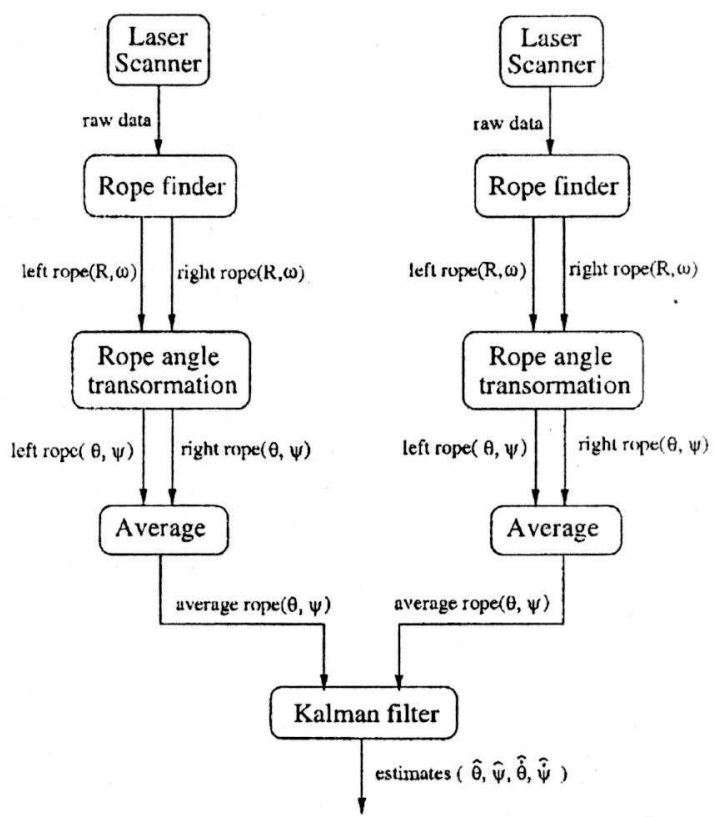

Fig. 6. Schematic of the hoist rope angle measurement system.

when the boom is moving through the air due to swinging, is unknown, and no data has yet been captured to indicate the scale of the problem. Some experiments were therefore carried out using an unmounted scanner at our laboratory in rain and in the open. The important measure, for the rope tracking system to work reliably, is the actual probability of seeing a rain drop in a given scan segment of the laser scanner, which for the two experiments conducted werc;

\begin{tabular}{cc} 
Rain rate & Probability (\%) \\
\hline 'light' & 0.02 \\
'medium' & 0.28
\end{tabular}

These probabilities are very low and therefore medium and light rain should not cause a significant problem. False targets may also be generated by insects and birds. Insects tend to swarm around the bright lights that are used to illuminate the pit at night.

4.1.1.2. Range gating The first stage of the rope finding algorithm is to range gate the raw laser scanner data. This means that all targets found between a range $R_{\min }$, and $R_{\max }$ are considered potential hoist rope targets. We can do this because we know that the ropes can only be in a certain region, which is constrained by the mechanical design. Figure 7 shows the region-of-interest created by these range gate limits combined with the restricted view from the sensor housing.

4.1.1.3. Rope discrimination (pairing data) For the dragline case where we are observing a pair of hoist ropes we have additional information that 


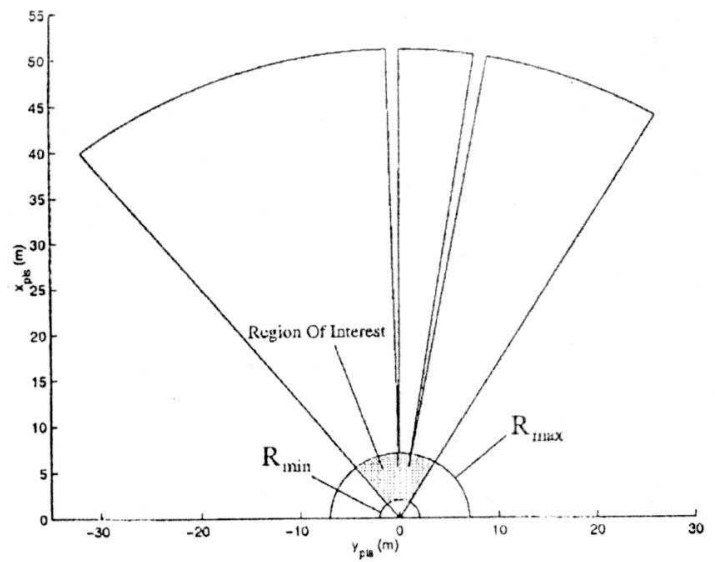

Fig. 7. Range data from a laser scanter showing the region-of-interest produced by range gating. The two large spikes indicate the position of the two hoist ropes.

can assist the discrimination process. This step would be skipped for a single hoist rope situation.

In particular we know that the ropes are constrained in the way they move relative to one another. This allows us to pair up data points into possible rope pairs (an $n^{2}$ search task). Experiinents have shown that the hoist ropes are always within $0.5 \mathrm{~m}$ of onc another in the $x$-direction and are between 0.5 and $1.0 \mathrm{~m}$ of one another in the $y$ direction. Figure 8 shows some data captured of the ropes when it was not raining, with some randomly added false target data added. The circles show the range gated data points. The squares show the mid-point between possible rope pairs. In Figure 8 three possible rope pairs can be seen.

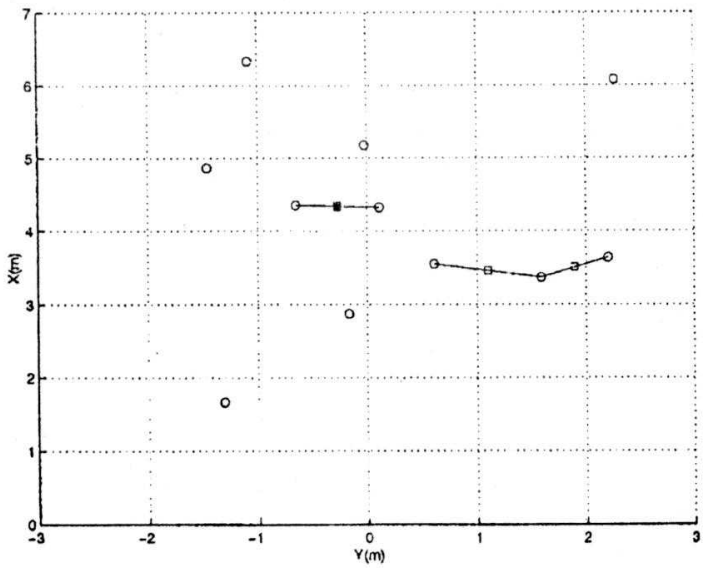

Fig. 8. Data from the scanner clearly showing paired datia points (squares) that represent the position of possible rope pairs.

4.1.2. Tracking and data association The next problem encountered is that of data association and tracking, i.e., how do we match pairs bctween successive scans, and hence identify the hoist ropes and reject the false target pairs? As observed by the laser scanners, which operate at a low frequency $(3 \mathrm{~Hz})$, the ropes move with an almost random walk type motion which makes directly tracking the hoist ropes using moclel based tracking techniques, such as Kalman filters, impossible.

The most practical (in this casc) and the simplest form of tracking and data association is to look for a target at time $t$ in the area immediately around where it was scen at time $t-1$. The size and shape of the scarch area is based on observed maximum motions between samples from experimental data.

The number of consecutive matches of a particular target pair can be counted and the pair with the highest count, assuming it is above some pre-defined threshold, should be the hoist, ropes (Figure 9).

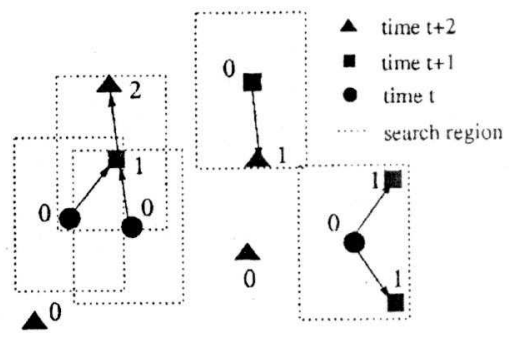

Fig. 9. The tracking and data association strategy. The numbers indicate the number of matches of each point (the search area is only shown for successful matches).

4.1.2.1. Rope modelling The position of the ropes found by the rope tracking systcm is with respect to the laser scanner. These positions must therefore be transformed into real swing and hoist angles of the hoist ropes. It is possible to derive an expression for both swing and hoist angles as a function of the sensed hoist rope position with respect to the laser scanner and the geometry of the sensing/boom structure. These transformations have been dcrived, but are not shown here.

\subsection{Kalman filtering}

The rope angle system consists of two laser scanners for robustness, and hence averaged swing and hoist angles are calculated for both scanners. A Kalman filter was used to fuse the measurements from the separate scanners, and to estimate the rate of change of swing and hoist angles, which are needed by the dragline control algorithm.

The angle data from each scanning system is not synchronous. The Kalman filter provides estimates at a fixed frequency $(5 \mathrm{H} /$ ) for the control 
system, whereas the measurements from the laser scanners arrive irregularly (on average $3 /$ sccond).

By its nature, the Kalman filter gives the covariance of the estimates which in turn can be used for two things:

(1) To reject a laser measurement if this measure is incompatible with the current estimate. In other words, it implements sensor failure detection.

(2) To validate the estimates. The estimates are valid once their covariance fall under a given threshold depending on the desired accuracy.

\subsection{Model of rope movement}

The model of the bucket/rope system is extremely simple. The swing angle $(\theta)$ is assumed to follow the equation of an un-forced pendulum as given in (3) and the hoist angle $(\dot{\psi})$ is assumed to follow a constant velocity $\ddot{\psi}=0$.

\subsection{Results}

The swing and hoist angle estimation system described here is currently being used in the prototype dragline swing automation system being trialled on a production dragline at a mine in Queensland, Australia. Figure 10 shows the results from the angle sensing system during a typical automatic cycle.
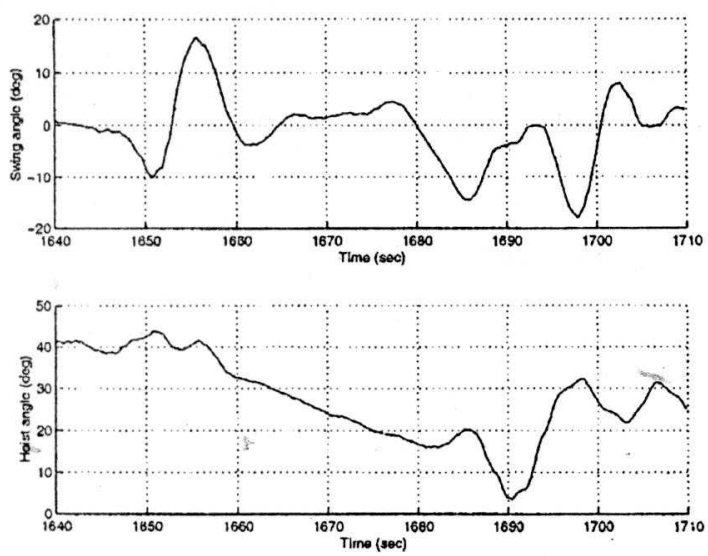

Fig. 10. Swing angle (top), and hoist angle (bottom) during an automatic cycle.

The swing and hoist angle system has so far been used to demonstrate:

- effective bucket swing damping at dumping and digging points;

- online estimation of bucket weight, which is used to determine when the bucket has emptied;

- bucket pose recovery.

\section{CONCLUSION}

We have presented an approach to the control of swinging loads based on feedback from online measurements of the load position. The sensing technique described does not contact the load or hoist rope yet is accurate and robust. The techniques described have been demonstrated in the control of a full scale production dragline, a Bucyrus-Erie 1370W.

\section{REFERENCES}

[1] A. Ridout, "Anti-swing control of the overhead crane using linear feedback," Journal of Electrical and Electronics Engineering, Australia, vol. 9, pp. 17-26, Mar. 1989.

[2] C. Backhouse, R.. Price, and P. Moore, "Crane automation," Industrial Robot, vol. 21, no. 4, pp. 22-25, 1994.

[3] J. Feddema, "Digital filter control of remotely operated flexible robotic structures," in Proc. American Control Conference, June 1993.

[4] R. DeSantis and S. Krau, "Bang bang control of a Cartesian crane," Robotica, vol. 12, pp. 449-454, 1994.

[5] A. Ridout, "Variably damped swing control, of the overhead crane," in Proc. 15th Annual Conf. IEEE Industrial Electronics Society, pp. 263-269, 1989.

[6] L. Tsitsilonis and D. McLean, "Stationkeeping control system for a helicopter with a suspended load," Trans Inst $M C$, vol. 3, pp. 121-134, July 1981.

[7] N. Gupta and A. Bryson, "Near-hover control of a helicopter with a hanging load," $J$. Aircraft, vol. 13, pp. 217-222, Mar. 1976.

[8] P. I. Corke, J. M. Roberts, and G. J. Winstanley, "Modelling and control of a 3500 tonne mining machine," in Experimental Robotics $V$ (A. Casals and A. de Almeida, eds.), no. 232 in Lecture Notes in Control and Information Sciences, pp. 262-274, Barcelona: Springer Verlag, June 1998.

[9] D. W. Hainsworth, P. I. Corke, and G. J. Winstanlcy, "Location of a dragline bucket in space using machine vision techniques," in Proc. Int. Conf. on Acoustics, Speech and Signal Processing (ICASSP-94), vol. 6, (Adelaide), pp. 161-164, Apr. 1994.

[10] G. Winstanley, D. Hainsworth, Y. Li, P. Corke, and H. Gurgenci, "Automatic control of dragline operation using machinc vision control of bucket position," in Proc. Int. Conf. on Mechatronics and Machine Vision in Practice, (Surfers Paradise), pp. 11-15, Sept. 1994. 